\title{
Mesoscopic transport of fermions through an engineered optical lattice connecting two reservoirs
}

\author{
M. Bruderer and W. Belzig \\ Fachbereich Physik, Universität Konstanz, D-78457 Konstanz, Germany
}

(Received 4 August 2011; published 17 January 2012)

\begin{abstract}
We study transport of fermions in a system composed of a short optical lattice connecting two finite atomic reservoirs at different filling levels. The average equilibration current through the optical lattice, for strong latticereservoir coupling and finite temperatures, is calculated within the Landauer formalism using a nonequilibrium Green's functions approach. We moreover determine quantum and thermal fluctuations in the transport and find significant shot-to-shot deviations from the average equilibration current. We show how to control the atomic current by engineering specific optical lattice potentials without requiring site-by-site manipulations and suggest the realization of a single level model. Based on this model we discuss the blocking effect on the atomic current resulting from weak interactions between the fermions.
\end{abstract}

DOI: 10.1103/PhysRevA.85.013623

PACS number(s): 67.85.-d, 05.60.Gg, 72.10.-d

\section{INTRODUCTION}

Ultracold atoms in optical lattices have been proven to be perfectly suited for implementing physical models of interest to the field of atomic and condensed matter physics [1,2]. Specifically, an important part of the related experimental efforts have improved and are still extending our understanding of nonequilibrium quantum transport. These efforts have resulted in the observation of fundamentally interesting quantum mechanical processes such as Bloch oscillations [3], Landau-Zener tunneling [4], and interaction-controlled transport [5]. Studying nonequilibrium transport in optical lattices has several advantages over conventional condensed matter systems: Ultracold atoms exhibit slow coherent quantum dynamics (with kilohertz tunneling rates) and are detectable in small numbers on microscopic scales. In addition, the ability to tune the interactions between atoms via Feshbach resonances makes it possible to investigate transport of interacting and noninteracting particles.

While many transport-related experiments employed tilted lattice potentials, alternative setups for studying nonequilibrium phenomena have been suggested, in which a current of particles flows between two atomic reservoirs. Micheli et al. [6-8] pointed out that, analogous to a transistor, an impurity atom may be utilized to control the flow of a one-dimensional Bose or Fermi gas. They considered the full time-dependent coherent dynamics of the ultracold gas in a closed system and determined the particle current by using analytical approximations [7] and time-dependent density matrix renormalization group (DMRG) calculations [8].

Pepino et al. $[9,10]$ generalized this idea by replacing the single impurity with an optical lattice coupled to separate bosonic reservoirs in order to emulate the behavior of semiconductor electronic circuits (see also [11]). In Refs. [9,10] reservoirs were introduced and specified as large sources or sinks of particles at zero temperature with a Fermi-sea-like energetic distribution, constant chemical potential, and fastdecaying system-reservoir correlations. A quantum master equation, relying on weak system-reservoir coupling, was used to describe time evolution of the system, thereby in part eliminating the coherent evolution of the reservoir.

Here we consider the evolution of a one-dimensional Fermi gas loaded into an optical lattice which is partitioned into

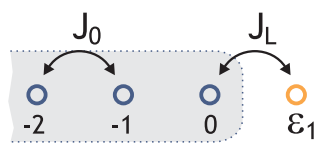

Reservoir L

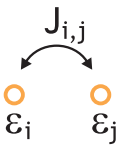

Coherent Region C

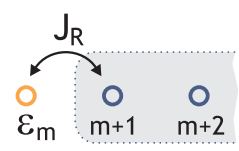

Reservoir $\mathrm{R}$
FIG. 1. (Color online) Fermions confined to an optical lattice hop from the left reservoir $L$ through a short coherent part $C$ (sites 1 to $m$ ) into the right reservoir $R$. The hopping parameters $J_{i, j}$, the on-site energies $\varepsilon_{j}$, and the couplings $J_{L}$ and $J_{R}$ may take arbitrarily engineered values, whereas the hopping parameter in the reservoirs $J_{0}$ is held constant.

two large incoherent reservoirs $L$ and $R$ connected by a short coherent region $C$, as illustrated in Fig. 1. In this setup the difference in the chemical potentials $\mu_{L}$ and $\mu_{R}$ of reservoirs $L$ and $R$ drives a current of fermionic atoms through the coherent region $C$.

In contrast to previous theoretical works, we take a mesoscopic perspective on nonequilibrium transport between the atomic reservoirs: We analyze the evolution of the Fermi gas within the Landauer formalism [12,13], where transport is described as a transmission process through the coherent region $C$ with fermions emitted and absorbed by the reservoirs $L$ and $R$, respectively. In this vein, we understand reservoirs to be finite containments filled with noninteracting fermions in the ground state, characterized by a Fermi distribution with finite temperature and well-defined (but not necessarily constant) chemical potential. The transmission is determined by a nonequilibrium Green's functions (NEGF) approach in the tight-binding picture $[14,15]$. This approach allows us to consider a wide range of experimentally relevant parameters. Moreover, we express our results in terms of the number of atoms accumulated in the reservoirs, which (in particular for small currents) may be more accessible in experiments than the current.

Accordingly, we complement and significantly extend the results in Refs. $[9,10]$ in several directions for the case of fermionic atoms: First, we take into account that experimental ultracold systems are finite and therefore we describe not only instantaneous steady-state currents at constant chemical potentials but the full equilibration process between the 
reservoirs. Second, our analysis is valid for arbitrarily engineered configurations of the coherent region $C$ and for strong coupling to the reservoirs, thereby going beyond the weak-coupling expansion in Refs. [9,10]. Strong coupling indeed corresponds to the most elementary setup with the parts $L, R$, and $C$ consisting of a single homogeneous optical lattice. Third, by using the tools of full counting statistics [13] and considering intrinsic damping mechanisms we explicitly evaluate the evolution of the fluctuations in the current. These fluctuations indicate large shot-to-shot deviations from the average value in repeated measurements of the number of particles in the reservoirs. Our approach includes finite-temperature effects relevant to both the average current and the fluctuations. For instance, thermal fluctuations are shown to build up during the equilibration process until they reach a constant value proportional to the temperature of the Fermi gas.

In the first part of this paper we analyze the evolution of the Fermi gas within the NEGF-Landauer formalism. In this framework we discuss the properties of the system in terms of the average current, the filling levels of the reservoirs, and fluctuations of these quantities. In the second part we first apply the formalism to the case of constant transmission between the reservoirs and subsequently discuss more complex situations. In particular, we show how to control the current by modulating the connecting optical lattice with the help of additional optical potentials. Finally, we suggest a way to realize a single-level model and demonstrate that in this model even weak interactions between the fermions are sufficient to reduce the atomic current.

\section{NEGF-LANDAUER MODEL}

We start with the theoretical framework required to determine the average equilibration current through the lattice and the filling levels of the reservoirs. The Hamiltonian of the system within the lowest Bloch band and in the tight-binding approximation is $\hat{H}_{C}+\hat{H}_{L}+\hat{H}_{R}+\hat{H}_{I}$ with

$$
\begin{aligned}
& \hat{H}_{C}=-\sum_{\langle i, j\rangle} J_{i, j} \hat{c}_{i}^{\dagger} \hat{c}_{j}+\sum_{j} \varepsilon_{j} \hat{c}_{j}^{\dagger} \hat{c}_{j}, \quad i, j \in 1, \ldots, m, \\
& \hat{H}_{L}=\hat{H}_{R}=-J_{0} \sum_{\langle i, j\rangle} \hat{c}_{i}^{\dagger} \hat{c}_{j}+\hat{H}_{S}, \quad i, j \notin 1, \ldots, m, \\
& \hat{H}_{I}=-J_{L}\left(\hat{c}_{1}^{\dagger} \hat{c}_{0}+\hat{c}_{0}^{\dagger} \hat{c}_{1}\right)-J_{R}\left(\hat{c}_{m}^{\dagger} \hat{c}_{m+1}+\hat{c}_{m+1}^{\dagger} \hat{c}_{m}\right),
\end{aligned}
$$

where the central part $C$ is formed by the sites 1 to $m$ and $\langle i, j\rangle$ denotes the sum over nearest neighbors. The operators $\hat{c}_{j}^{\dagger}\left(\hat{c}_{j}\right)$ create (annihilate) a spin-polarized fermion in a Wannier state localized at site $j$. The hopping parameters in the central part and in the reservoirs are $J_{i, j}$ and $J_{0}$, respectively, and $\varepsilon_{j}$ are on-site energies. The couplings $J_{L}$ and $J_{R}$ connect the central part to the reservoirs which are each composed of $M$ sites.

The Hamiltonian $\hat{H}_{S}$, not specified explicitly, represents interactions of the reservoirs with an engineered environment, e.g., with an atomic gas or optical radiation that is not necessarily far detuned. The interactions introduced by $\hat{H}_{S}$ add dissipative and incoherent processes to the reservoirs so that they act as semiclassical systems equivalent to metallic electrodes. These processes are assumed to destroy coherence and to relax the fermions to the ground state on a time scale shorter than $\hbar / J_{0}$, making it possible to attribute a Fermi distribution with well-defined temperature and chemical potential to the reservoirs. An explicit scheme suggested in Ref. [16] achieves this aim through a combination of coherent laser excitations and dissipation into an ambient superfluid. First, fermions with high momentum are transferred into the first excited band of the optical lattice via a Raman process. Subsequently, the excited states decay into the lowest Bloch band due to emission of phonons into the superfluid. An iteration of this procedure results in a stable Fermi distribution of the atoms in the reservoirs.

To apply the Landauer formalism modified to account for the finite size of the reservoirs, we introduce the number operators $\hat{N}_{\alpha}$, with $\alpha=L$ or $R$, measuring the number of fermions in the reservoirs and the expectation values $\left\langle\hat{N}_{\alpha}\right\rangle$. We specify the state of the system by the average particle number $\left\langle\hat{N}_{\alpha}\right\rangle$ and the current $\partial_{t}\left\langle\hat{N}_{\alpha}\right\rangle$ through the central part. To obtain the current in the Landauer formalism we treat the connecting optical lattice as a scattering potential with the energy-dependent transmission $\mathcal{T}(\varepsilon)$. Hence the average current $\partial_{t}\left\langle\hat{N}_{R}\right\rangle=-\partial_{t}\left\langle\hat{N}_{L}\right\rangle$ is the sum of all possible scattering transfers between the two reservoirs

$$
\partial_{t}\left\langle\hat{N}_{R}(t)\right\rangle=\int \frac{d \varepsilon}{2 \pi \hbar} \mathcal{T}(\varepsilon)\left[f_{L}(\varepsilon, t)-f_{R}(\varepsilon, t)\right] .
$$

The Fermi functions of the reservoirs are $f_{\alpha}(\varepsilon, t)=$ $\left[e^{\left(\varepsilon-\mu_{\alpha}\right) / k_{B} T}+1\right]^{-1}$, with the Boltzmann constant $k_{B}$, the temperature $T$, and the time-dependent chemical potential $\mu_{\alpha}(t)$. Note that Eq. (2) is approximately valid provided that $f_{\alpha}(\varepsilon, t)$, or equivalently $\mu_{\alpha}(t)$, varies slowly on the microscopic time scale $\hbar / J_{0}$. This can readily be achieved by either increasing the size of the reservoirs $M$ or decreasing the transmission through the central part $C$.

The chemical potential $\mu_{\alpha}$ is related to the particle number $\left\langle\hat{N}_{\alpha}\right\rangle$ since the reservoirs are finite and the total number of fermions in the system is fixed. The implicit relation between $\mu_{\alpha}$ and the particle number $\left\langle\hat{N}_{\alpha}\right\rangle$ is

$$
\left\langle\hat{N}_{\alpha}\right\rangle=M \int d \varepsilon \rho(\varepsilon) f_{\alpha}(\varepsilon),
$$

with the density of states of the reservoir given by $\rho(\varepsilon)=$ $1 / \pi \sqrt{\left(2 J_{0}\right)^{2}-\left(\varepsilon-2 J_{0}\right)^{2}}$ for $0 \leqslant \varepsilon \leqslant 4 J_{0}$ and zero otherwise. Upon solving Eq. (3) for $\mu_{\alpha}(t)$, either analytically or numerically, one finds the chemical potential $\mu_{\alpha}(t)$ as a function of $\left\langle\hat{N}_{\alpha}(t)\right\rangle$. Thus, as a consequence of the finite-size reservoirs the Landauer formula in Eq. (2) becomes a closed (integro-differential) equation for $\left\langle\hat{N}_{\alpha}(t)\right\rangle$.

The transmission $\mathcal{T}(\varepsilon)$ is efficiently determined by use of the NEGF approach $[14,15]$. In terms of Green's functions we have

$$
\mathcal{T}(\varepsilon)=\left|G_{1, m}(\varepsilon)\right|^{2} \Gamma_{L}(\varepsilon) \Gamma_{R}(\varepsilon),
$$

where $G_{i, j}(\varepsilon)$ is the full retarded Green's function for the central part and $\Gamma_{\alpha}(\varepsilon)=2 \pi J_{\alpha}^{2} \rho_{\alpha}(\varepsilon)$ describes the coupling to the reservoirs. Here, $\rho_{L}(\varepsilon)$ and $\rho_{R}(\varepsilon)$ are, respectively, the local density of states at sites 0 and $m+1$ (see Fig. 1). Consequently $\mathcal{T}(\varepsilon)$ encodes the coherent evolution of the central part, governed by $\hat{H}_{C}$, as well as the coupling to the reservoirs. 
To calculate the full Green's function $G_{i, j}(\varepsilon)$ from $\hat{H}_{C}$ we start with the bare Green's function $g_{i, j}(\varepsilon)$ whose components obey the equation

$$
\left(\varepsilon-\varepsilon_{i}\right) g_{i, j}+\sum_{k} J_{i, k} g_{k, j}=\delta_{i, j}
$$

or equivalently $g(\varepsilon)=1 /\left(\varepsilon-H_{C}\right)$, with both the Green's function and $H_{C}$ in matrix notation. The bare Green's function $g_{i, j}(\varepsilon)$ with poles at the energy levels of $H_{C}$ would be sufficient to determine the transmission if the coherent region $C$ were coupled weakly to the reservoirs. However, the full propagation of a fermion between the sites $i$ and $j$ includes excursions into the reservoirs due to the coupling, which leads to the broadening $\Gamma_{\alpha} \sim J_{\alpha}^{2} / J_{0}$ of the energy levels. More precisely, the excursions result in corrections to $g_{i, j}(\varepsilon)$ in terms of self-energies $\Sigma_{L}=J_{L}^{2} \tilde{g}_{0,0}$ and $\Sigma_{R}=J_{R}^{2} \tilde{g}_{m+1, m+1}$, where $\tilde{g}_{i, j}(\varepsilon)$ denote the Green's functions of the reservoirs. Including the coupling to the reservoirs to all orders we arrive at the Dyson equation for the full Green's function

$$
G_{i, j}=g_{i, j}+g_{i, 1} \Sigma_{L} G_{1, j}+g_{i, m} \Sigma_{R} G_{m, j} .
$$

The relevant matrix element for the transmission $G_{1, m}$ is then obtained from Eq. (5) by solving a set of simultaneous equations for $G_{1,1}, G_{m, m}, G_{1, m}$, and $G_{m, 1}$. As a result one finds $G_{1, m}=g_{1, m} / D$ with [14]

$$
D=\left(1-\Sigma_{L} g_{1,1}\right)\left(1-\Sigma_{R} g_{m, m}\right)-\Sigma_{L} \Sigma_{R} g_{1, m}^{2}
$$

assuming that $g_{1, m}=g_{m, 1}$.

For our specific setup we treat the reservoirs as semi-infinite optical lattices, for which the Green's function $\tilde{g}_{i, j}$ at the end site 0 reads [15]

$$
\tilde{g}_{0,0}(\varepsilon)=\left[\left(\varepsilon-2 J_{0}\right)-i \sqrt{\left(2 J_{0}\right)^{2}-\left(\varepsilon-2 J_{0}\right)^{2}}\right] / 2 J_{0}^{2}
$$

and $\tilde{g}_{m+1, m+1}=\tilde{g}_{0,0}$; the local density of states is given by $\rho_{\alpha}(\varepsilon)=-(1 / \pi) \operatorname{Im} \tilde{g}_{0,0}(\varepsilon)$. These relations allow us to find explicit expressions for the couplings $\Gamma_{\alpha}$ and the self-energies $\Sigma_{\alpha}$ in Eq. (5).

\section{FLUCTUATIONS AND DAMPING}

So far our analysis was restricted to the average fermion number $\left\langle\hat{N}_{\alpha}(t)\right\rangle$ and the current $\partial_{t}\left\langle\hat{N}_{\alpha}(t)\right\rangle$, which are found from Eqs. (2) and (3) for a given transmission $\mathcal{T}(\varepsilon)$ in Eq. (4). We now turn our attention to quantum and thermal fluctuations present in the system. To simplify the problem of determining the fluctuations we treat creation and damping processes separately and, if possible, add their effects together.

Fluctuations created during the evolution of the system can be found by using the Levitov formula [13], which yields the full counting statistics of thermal and quantum fluctuations provided $\left\langle\hat{N}_{\alpha}(t)\right\rangle$ and hence $\mu_{\alpha}(t)$ are known. However, we limit our analysis to the most relevant statistical parameter, namely the variance of the number of fermions $\left\langle\delta N_{\alpha}^{2}\right\rangle \equiv$ $\left\langle\left(\hat{N}_{\alpha}-\left\langle\hat{N}_{\alpha}\right\rangle\right)^{2}\right\rangle$. With initially no fluctuations present we find for the variance $\left\langle\delta N_{\alpha}^{2}(t)\right\rangle$ after a time $t$ according to the Levitov formula

$$
\begin{aligned}
\left\langle\delta N_{\alpha}^{2}(t)\right\rangle= & \int_{0}^{t} d s \int \frac{d \varepsilon}{2 \pi \hbar}\left\{\mathcal{T}(\varepsilon) \overline{\mathcal{T}}(\varepsilon)\left[f_{L}(\varepsilon, s)-f_{R}(\varepsilon, s)\right]^{2}\right. \\
& \left.+\mathcal{T}(\varepsilon)\left[f_{L}(\varepsilon, s) \bar{f}_{L}(\varepsilon, s)+f_{R}(\varepsilon, s) \bar{f}_{R}(\varepsilon, s)\right]\right\},
\end{aligned}
$$

where we introduced $\bar{f}_{\alpha}(\varepsilon, t)=1-f_{\alpha}(\varepsilon, t)$ and $\overline{\mathcal{T}}(\varepsilon)=1-$ $\mathcal{T}(\varepsilon)$. At zero temperature, Eq. (6) describes the creation of quantum fluctuations caused by the probabilistic nature of the particle transfer through the optical lattice. On the other hand, at equilibrium between the reservoirs and for $\mathcal{T}(\varepsilon) \equiv 1$ the fluctuations are purely thermal.

Unlike in conventional mesoscopic systems with infinitesized electrodes, the fluctuations described by Eq. (6) are constantly damped out at a rate $\gamma$. This intrinsic damping occurs because fluctuations in the current immediately lead to fluctuations of the chemical potentials, which drive the system back to the evolution according to the mean-field description $\left\langle\hat{N}_{\alpha}(t)\right\rangle$. To find an explicit expression for the intrinsic damping $\gamma$ we determine the change in the chemical potential $\delta \mu_{\alpha}$ caused by an excess of particles $\delta N_{\alpha} \ll\left\langle\hat{N}_{\alpha}\right\rangle$ with respect to $\left\langle\hat{N}_{\alpha}\right\rangle$. From Eq. (3) we obtain $\left\langle\hat{N}_{\alpha}\right\rangle+\delta N_{\alpha}=$ $M \int d \varepsilon \rho(\varepsilon) f_{\alpha}\left(\varepsilon, \mu_{\alpha}+\delta \mu_{\alpha}\right)$, which to lowest order in $\delta \mu_{\alpha}$ and $\delta N_{\alpha}$ results in the linear dependence $\delta \mu_{\alpha}=\delta N_{\alpha} / M \rho_{T}\left(\mu_{\alpha}\right)$ with

$$
\rho_{T}\left(\mu_{\alpha}\right)=\frac{1}{4 k_{B} T} \int d \varepsilon \rho(\varepsilon) \operatorname{sech}^{2}\left(\frac{\varepsilon-\mu_{\alpha}}{2 k_{B} T}\right) .
$$

Note that $\rho_{T}\left(\mu_{\alpha}\right)$ reduces to $\rho\left(\mu_{\alpha}\right)$ in the case $T=0$ and to $k_{B} T / 4$ in the limit of infinite temperature. Similarly, we expand the Landauer formula to lowest order in $\delta \mu_{\alpha}$ and $\partial_{t}\left(\delta N_{\alpha}\right)$ and use the relation $\delta \mu_{\alpha}=\delta N_{\alpha} / M \rho_{T}\left(\mu_{\alpha}\right)$ to find the time dependence for small fluctuations $\partial_{t}\left(\delta N_{\alpha}\right)=-\gamma \delta N_{\alpha}$ with the damping factor

$$
\gamma=\frac{1}{2 \pi \hbar M}\left[\frac{\mathcal{T}_{T}\left(\mu_{L}\right)}{\rho_{T}\left(\mu_{L}\right)}+\frac{\mathcal{T}_{T}\left(\mu_{R}\right)}{\rho_{T}\left(\mu_{R}\right)}\right],
$$

where $\mathcal{T}_{T}\left(\mu_{\alpha}\right)$ is defined in the same way as $\rho_{T}\left(\mu_{\alpha}\right)$ in Eq. (7). The factor $\gamma$ depends on the filling level of the reservoirs and is therefore time dependent in general. Importantly, $\gamma$ is always positive and thus fluctuations are indeed damped out.

As a result, the fluctuations in the system depend not only on the properties of the coherent region but also on the reservoirs via their density of states $\rho(\varepsilon)$ evaluated at the filling level. Thus by choosing the appropriate reservoirs it should be possible to perform experiments in either the fluctuation-dominated or the mean-field regime. This works particularly well for an optical lattice reservoir since its density of states varies considerably over the entire bandwidth $4 J_{0}$ so that the intrinsic damping $\gamma$ can be tuned over a wide range.

\section{CONSTANT TRANSMISSION}

To gain physical insight into the transport between reservoirs we apply our general results to the important special case of constant transmission: $\mathcal{T}(\varepsilon) \equiv \mathcal{T}_{0}$ for $0 \leqslant \varepsilon \leqslant 4 J_{0}$ and zero otherwise, where the constant transmission $\mathcal{T}_{0}$ takes values $0 \leqslant \mathcal{T}_{0} \leqslant 1$. In this case the Landauer formula for the current reduces to $\partial_{t}\left\langle\hat{N}_{R}\right\rangle=\mathcal{T}_{0} \Delta \mu / 2 \pi \hbar$ and hence one only has to determine the dependence of $\Delta \mu=\mu_{L}-\mu_{R}$ on the average particle number $\left\langle\hat{N}_{\alpha}\right\rangle$ and the temperature $T$.

For concreteness we consider the equilibration process in the low-temperature regime $k_{B} T \ll J_{0}$, neglecting corrections of the order $\left(k_{B} T / J_{0}\right)^{2}$. In this case the chemical potential difference is $\Delta \mu=4 J_{0} \cos \left(\pi\left\langle\hat{N}_{R}\right\rangle / M\right)$ assuming the initial 
conditions $\left\langle\hat{N}_{L}\right\rangle=M$ and $\left\langle\hat{N}_{R}\right\rangle=0$. Solving the Landauer equation we obtain the evolution of the filling level

$$
\left\langle\hat{N}_{R}(t)\right\rangle=C U \arctan \left[\sinh \left(t / t_{e q}\right)\right]
$$

and the current through the central region $\partial_{t}\left\langle\hat{N}_{R}(t)\right\rangle=$ $(U / R) \operatorname{sech}\left(t / t_{e q}\right)$. The equilibration time scale $t_{e q}=R C$ satisfies $t_{e q} \gg \hbar / J_{0}$ in the relevant parameter regime as required for Eq. (2) to be valid. Here, analogous to a classical RC circuit, we introduced the resistance $R=2 \pi \hbar / \mathcal{T}_{0}$, the capacitance $C=M / 4 \pi J_{0}$, and initial capacitor voltage $U=4 J_{0}$.

We next determine the evolution of the fluctuations during the equilibration process. For simplicity we assume from now on that $\mathcal{T}_{0}$ is close to unity, i.e., $\mathcal{T}_{0} \approx 1$, so that predominantly thermal fluctuations with $\left\langle\delta N_{\alpha}^{2}(t)\right\rangle=2 t k_{B} T / R$ are created during the equilibration process, as can be found from the Levitov formula in Eq. (6). Since correlations of thermal fluctuations decay fast on the time scale $t_{e q}$, the evolution of the deviations $\delta N_{R}$ from $\left\langle\hat{N}_{R}(t)\right\rangle$ can be expressed in the form of a Langevin equation

$$
\partial_{t}\left(\delta N_{R}\right)=-\gamma(t) \delta N_{R}+\xi(t) .
$$

The time-dependent damping factor resulting from Eq. (8) reads $\gamma(t)=\sin \left[\pi\left\langle\hat{N}_{R}(t)\right\rangle / M\right] / R C$ and thus damping increases as the system approaches equilibrium. The fluctuations are represented by the stochastic force $\xi(t)$ and satisfy the condition $\left\langle\xi(t) \xi\left(t^{\prime}\right)\right\rangle=\left(2 k_{B} T / R\right) \delta\left(t-t^{\prime}\right)$. Using standard techniques for stochastic problems [17] we find the evolution of the fluctuations

$$
\left\langle\delta N_{R}^{2}(t)\right\rangle=C k_{B} T\left[\operatorname{sech}^{2}\left(t / t_{e q}\right) t / t_{e q}+\tanh \left(t / t_{e q}\right)\right] .
$$

We see that the fluctuations increase linearly in the regime $t / t_{e q} \ll 1$, where damping is weak according to Eq. (8). On the other hand, in the limit $t / t_{e q} \gg 1$ the fluctuations converge to the constant value $C k_{B} T$, which results from the competition between thermal fluctuations and the intrinsic damping $\gamma=$ $1 / R C$ at equilibrium.

Figure 2 shows the time evolution of the average filling level $\left\langle\hat{N}_{R}(t)\right\rangle / M$ and the fluctuations in the right reservoir. The filling level increases linearly in the regime $t / t_{e q} \ll 1$ and saturates at the equilibrium value $\left\langle\hat{N}_{R}\right\rangle=\left\langle\hat{N}_{L}\right\rangle=M / 2$. Thermal fluctuations around the average are limited by damping; however, they indicate significant shot-to-shot deviations from the average filling level. Of particular interest is the Fano factor defined by $F=\left\langle\delta N_{R}^{2}(t)\right\rangle /\left\langle\hat{N}_{R}(t)\right\rangle$, which is independent of the size of the reservoirs $M$ and gives a direct measure of the temperature of the system. The Fano factor converges to the value $F=k_{B} T / 2 \pi J_{0}$ as the system equilibrates, i.e., in the limit $t / t_{e q} \gg 1$. This result even holds for arbitrary constant transmissions $\mathcal{T}_{0}$ as quantum fluctuations are damped out in this limit.

An elementary experimental configuration with constant transmission, namely $\mathcal{T}_{0} \approx 1$, consists of a single homogeneous optical lattice partitioned into a sufficiently long coherent part $C$ and the reservoirs $L$ and $R$. The corresponding hopping parameters and couplings are $J_{i, j}=J_{0}$ and $J_{\alpha}=J_{0}$, respectively. In fact, the strong couplings $J_{\alpha}$ lead to significant broadening of the cosine-distributed energy levels of the coherent part [18]. As a consequence, the broadened energy

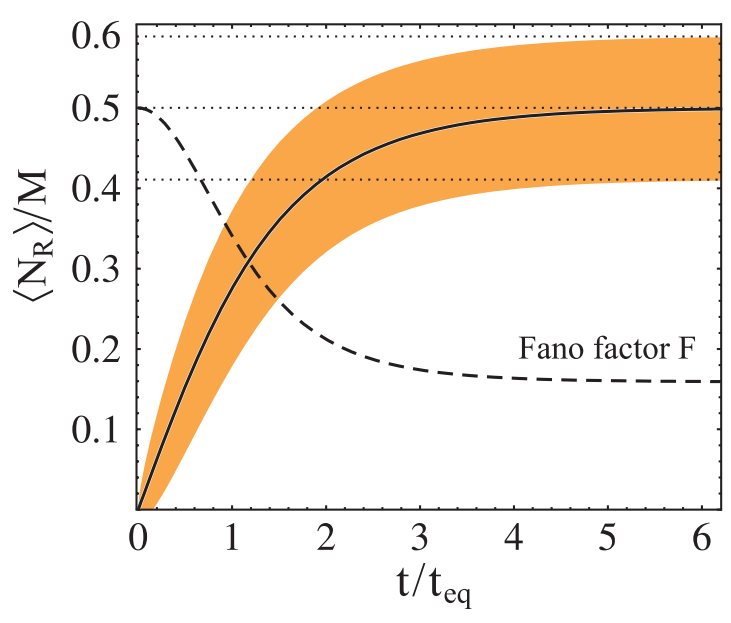

FIG. 2. (Color online) Filling level and fluctuations in the right reservoir (initially empty) for a constant transmission $\mathcal{T}_{0}$. The average filling level $\left\langle N_{R}(t)\right\rangle / M$ (solid line) increases with time $t / t_{e q}$ and saturates at the equilibrium value $1 / 2$. The standard deviation $\sqrt{\left\langle\delta N_{R}^{2}(t)\right\rangle} / M$ from the average, due to thermal fluctuations, is indicated by the orange (gray) band. The Fano factor $F=$ $\left\langle\delta N_{R}^{2}(t)\right\rangle /\left\langle N_{R}(t)\right\rangle$ (dashed line) decreases with time and approaches a constant value in the regime $t / t_{e q} \gg 1$. The parameters are $k_{B} T / J_{0} M=1 / 10$ for the filling level and $k_{B} T / J_{0}=1$ for the Fano factor.

levels merge together in the regime $J_{\alpha} \gg 2 \pi J_{0} / m$, which results in an approximately constant transmission.

\section{TRANSMISSION ENGINEERING}

The usefulness of the NEGF-Landauer formalism is most evident if we want to calculate the current through an engineered optical lattice with tailored parameters $J_{i, j}$ and $\varepsilon_{j}$. Since the optical lattice potentials for the reservoirs and the central region would most likely be produced by the same counterpropagating laser beams we set $J_{i, j}=J_{\alpha}=J_{0}$ and focus on modified on-site energies $\varepsilon_{j}$. A possible experimental configuration with tailored on-site energies $\varepsilon_{j}$ involves one (or several) laser beams crossing the central region perpendicular to the optical lattice. The optical potential caused by a single beam centered at position $v$ shifts the energies as

$$
\varepsilon_{j}=V \exp \left[-(j-v)^{2} / \sigma^{2}\right],
$$

with the potential strength $V$ and width $\sigma$ measured in units of lattice spacings. Depending on the detuning, $V$ may take positive or negative values [19]. We emphasize that unlike the scheme proposed in Ref. [9] such a configuration does not require site-by-site control of the optical lattice, neither of the on-site energies $\varepsilon_{j}$ nor the hopping parameters $J_{i, j}$ or $J_{\alpha}$.

A specific setup consists of a single laser beam, centered at $v \approx m / 2$ and with beam waist $\sigma \sim m / 4$, acting as a $V$-dependent switch for the fermion current. The potential shifts the energies $\varepsilon_{j}$ out of the reservoir band and thus reduces the transmission significantly, as shown in Fig. 3(a). This configuration provides the possibility to study the dependence of the fluctuations on the transmission $\mathcal{T}(\varepsilon)$ and the temperature $T$ of the fermions: If we choose the initial conditions $\left\langle\hat{N}_{L}\right\rangle=M,\left\langle\hat{N}_{R}\right\rangle=0$ and stay far from 

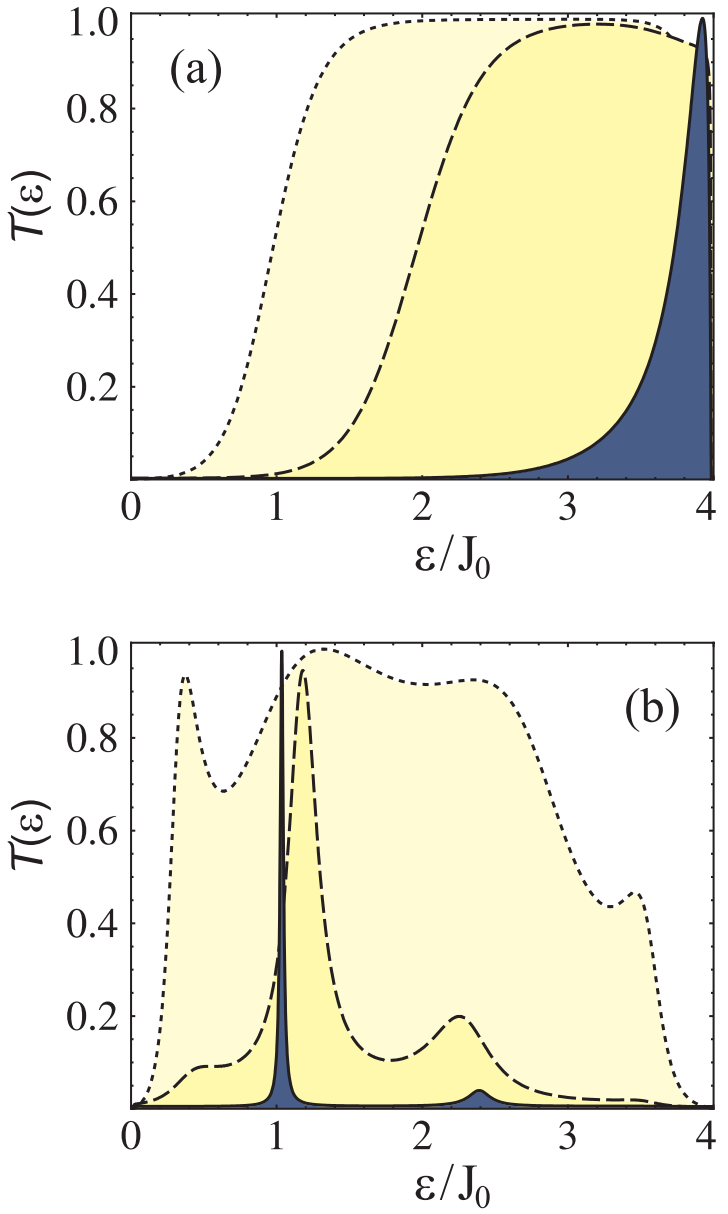

FIG. 3. (Color online) The engineered transmission $\mathcal{T}(\varepsilon)$ as a function of the energy $\varepsilon / J_{0}$ for different modulations of an optical lattice with length $m=10$. (a) A single centered beam with waist $\sigma=2$ and increasing depths $V / J_{0}=1,2,4$ (dotted, dashed, and full line) shifts the energies out of the reservoir band and reduces the transmission to a peak at the upper band limit. (b) Two beams isolate a few central lattice sites and create a single resonant level at $\varepsilon / J_{0} \approx 1$ for sufficiently strong intensities. The beams are positioned at $v_{1}=3$, $\nu_{2}=8$ with waist $\sigma=1$, depths $V_{2}=1,3,5$ (dotted, dashed, and full line) and $V_{1} / V_{2}=1 / 2$.

equilibrium then according to Eq. (8) damping is negligible. As a consequence, thermal and quantum fluctuations lead to significant deviations of the filling level from $\left\langle\hat{N}_{R}(t)\right\rangle$, which are detectable by counting the actual number of fermions in the right reservoir. Figure 4 shows the average particle number $\left\langle\hat{N}_{R}(t)\right\rangle$ and the expected fluctuations for modulations of the optical lattice with two different potential strengths $V$. At zero temperature, only quantum fluctuations caused by the limited transmission contribute, whereas at finite temperature fluctuations are further increased. By comparing Figs. 4(a) and 4(b) we see that quantum fluctuations become more important for reduced transmissions, i.e., for stronger potential strengths $V$.

A paradigmatic system in the context of mesoscopic physics is the single-level model [15], or in the case of interacting fermions the Anderson impurity model [20]. These models may be used, e.g., to study the Kondo effect or to describe transport through a single quantum dot. Realization of a single-
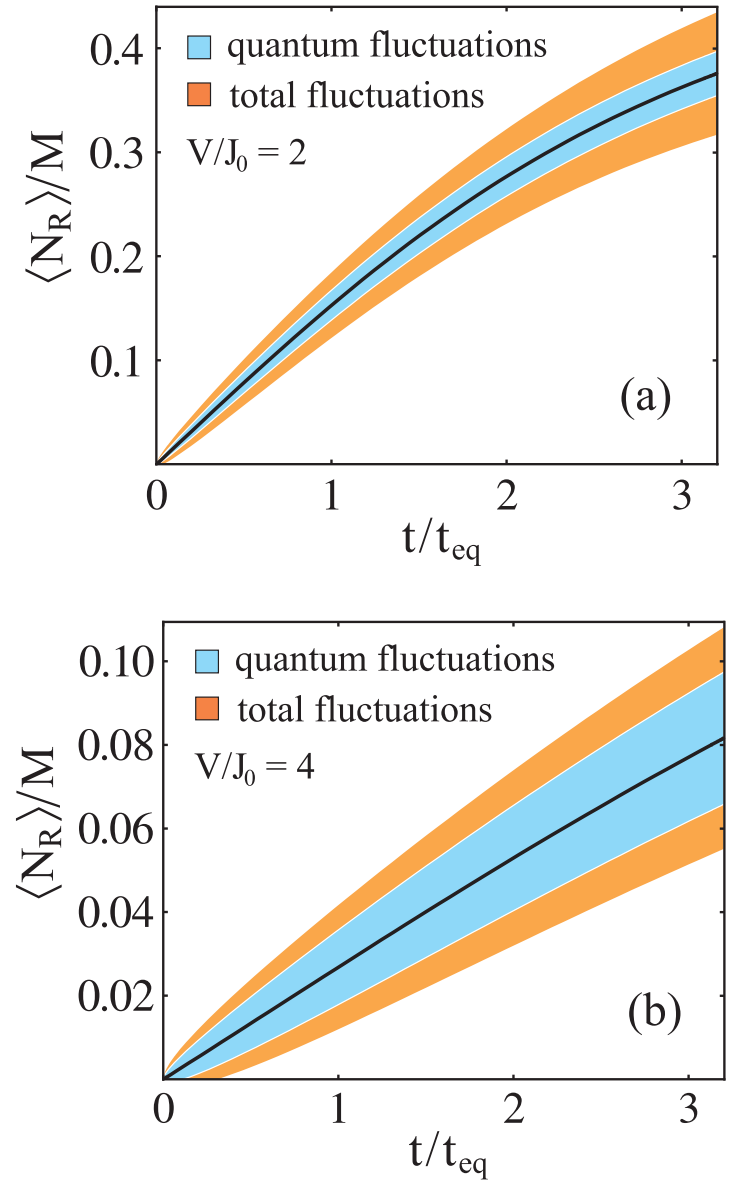

FIG. 4. (Color online) Filling level and fluctuations in the right reservoir (initially empty) for a lattice modulated by a single beam. (a) The average filling level $\left\langle N_{R}(t)\right\rangle / M$ (solid line) as a function time $t / t_{e q}$ (with $t_{e q}=\hbar M / 2 J_{0}$ ) for the modulation strength $V / J_{0}=2$. Different bands indicate the standard deviation $\sqrt{\left\langle\delta N_{R}^{2}(t)\right\rangle} / M$ due to quantum fluctuations (blue or light gray) and total fluctuations, i.e., quantum plus thermal (orange or dark gray). (b) The same quantities for a stronger modulation strength $V / J_{0}=4$. The lower transmission results in a smaller current between the reservoirs and enhanced quantum fluctuations in comparison to (a). In both plots damping has not been taken into account and the parameters are $k_{B} T / J_{0}=1$, $m=10$, and $M=50$.

level model can be achieved by means of two laser beams with different detuning leading to energy shifts

$$
\varepsilon_{j}=V_{1} \exp \left[-\left(j-v_{1}\right)^{2} / \sigma^{2}\right]-V_{2} \exp \left[-\left(j-v_{2}\right)^{2} / \sigma^{2}\right] .
$$

If the beams are separated with $v_{1}-v_{2} \sim m / 2$ and narrow $\sigma \sim m / 4$ then for sufficiently strong potentials $V_{1}, V_{2}>0$ the transmission exhibits a single peak, as shown in Fig. 3(b). The position of the peak depends on the ratio $V_{1} / V_{2}$ and the strength of the potentials determines the width of the peak, i.e., the effective coupling to the reservoirs.

The emergence of the single level can be understood in the energy band picture: The first beam shifts the unmodulated band of width $4 J_{0}$ upward, while the second beam shifts the band downward. As a consequence, a small region between the beams is isolated from the reservoirs and acts as a single energy level. The effective couplings $\Gamma_{\alpha}$ of the level to the 
reservoirs, or equivalently the width of the single level, is readily controllable by the strengths of the beams $V_{1}$ and $V_{2}$. This makes it possible to access the weak-coupling regime considered in Ref. [9] as well as the strong-coupling regime without the requirement of specific control of the hopping parameters $J_{\alpha}$.

\section{EFFECT OF INTERACTIONS}

Let us now discuss the effect of weak interactions between the fermions on the basis of the single-level model. If we consider a spin-balanced mixture of fermions in two different internal states, denoted by up and down, then the total Hamiltonian in Eq. (1) is augmented by the interaction term $\hat{H}_{U}=U \sum_{j} \hat{n}_{j}^{\uparrow} \hat{n}_{j}^{\downarrow}$. Here, $U$ is the either positive or negative interaction strength between the fermions and $\hat{n}_{j}^{\uparrow}\left(\hat{n}_{j}^{\downarrow}\right)$ is the occupation number operator for the up (down) states. For our analysis we use the mean-field approximation $\hat{n}_{j}^{\uparrow} \hat{n}_{j}^{\downarrow} \approx \hat{n}_{j}^{\uparrow}\left\langle\hat{n}_{j}^{\downarrow}\right\rangle+\left\langle\hat{n}_{j}^{\uparrow}\right\rangle \hat{n}_{j}^{\downarrow}-\left\langle\hat{n}_{j}^{\uparrow}\right\rangle\left\langle\hat{n}_{j}^{\downarrow}\right\rangle$ which we expect to be valid for sufficiently small reservoirs and low filling levels [21]. The chemical potentials of the reservoirs $\mu_{\alpha}$ are changed accordingly due to the interactions; e.g., the chemical potential for spin-up states is given by $\mu_{\alpha}^{\uparrow}=\mu_{\alpha}+U\left\langle\hat{n}^{\downarrow}\right\rangle$. In the following we will however neglect these trivial changes.

The effect of interactions on the single level is to shift its original $(U=0)$ position $\varepsilon_{0}$ by the interaction energy; e.g., for spin-up fermions the level position is $\varepsilon_{0}+U\left\langle\hat{n}^{\downarrow}\right\rangle$. The corresponding Green's function reads

$$
G^{\uparrow}(\varepsilon)=1 /\left[\varepsilon-\varepsilon_{0}-U\left\langle\hat{n}^{\downarrow}\right\rangle+i \Gamma_{L}(\varepsilon)+i \Gamma_{R}(\varepsilon)\right]
$$

with the effective couplings $\Gamma_{\alpha}$. The occupation of the single level is given by

$$
\left\langle\hat{n}^{\uparrow}\right\rangle=\int \frac{d \varepsilon}{2 \pi}\left|G^{\uparrow}(\varepsilon)\right|^{2}\left[\Gamma_{L}(\varepsilon) f_{L}(\varepsilon)+\Gamma_{R}(\varepsilon) f_{R}(\varepsilon)\right],
$$

with the time dependence of the Fermi functions omitted. In the case of a spin-balanced mixture with $\left\langle\hat{n}^{\uparrow}\right\rangle=\left\langle\hat{n}^{\downarrow}\right\rangle$ the Green's function $G^{\uparrow}(\varepsilon)$ and the occupation $\left\langle\hat{n}^{\uparrow}\right\rangle$ can be determined self-consistently to obtain the transmission $\mathcal{T}(\varepsilon) \propto\left|G^{\uparrow}(\varepsilon)\right|^{2}$, which is the same for both internal states. In principle, the average particle number $\left\langle\hat{N}_{\alpha}(t)\right\rangle$ and the current $\partial_{t}\left\langle\hat{N}_{\alpha}(t)\right\rangle$ are then evaluated as for noninteracting fermions.

The effect of interactions on the transmission can be qualitatively understood in the wide-band limit with constant couplings $\Gamma_{\alpha}=\Gamma$. Inserting $G^{\uparrow}(\varepsilon)$ into Eq. (10) then yields the self-consistent equation for the occupation [20]

$$
\langle\hat{n}\rangle=\frac{1}{2}+\frac{2}{\pi} \sum_{\alpha} \arctan \left(\frac{\mu_{\alpha}-\varepsilon_{0}-U\langle\hat{n}\rangle}{2 \Gamma}\right),
$$

where the average occupation number applies to both spin states; i.e., $\langle\hat{n}\rangle=\left\langle\hat{n}^{\uparrow}\right\rangle=\left\langle\hat{n}^{\downarrow}\right\rangle$. Figure 5 shows the average occupation $\langle\hat{n}\rangle$ of the single level and the energy shift $U\langle\hat{n}\rangle$ as a function of the interaction strength $U$ according to Eq. (11). For repulsive interactions $U>0$ we observe a depletion of the single level and a shift to higher energies. This shift is bounded by $\mu_{L}$ in the limit of vanishing coupling $\Gamma$, but takes values larger than $\mu_{L}$ in the case of finite $\Gamma$. For attractive interactions $U<0$ the occupation $\langle\hat{n}\rangle$ increases and makes an abrupt transition to $\langle\hat{n}\rangle \approx 1$ accompanied by a shift of the

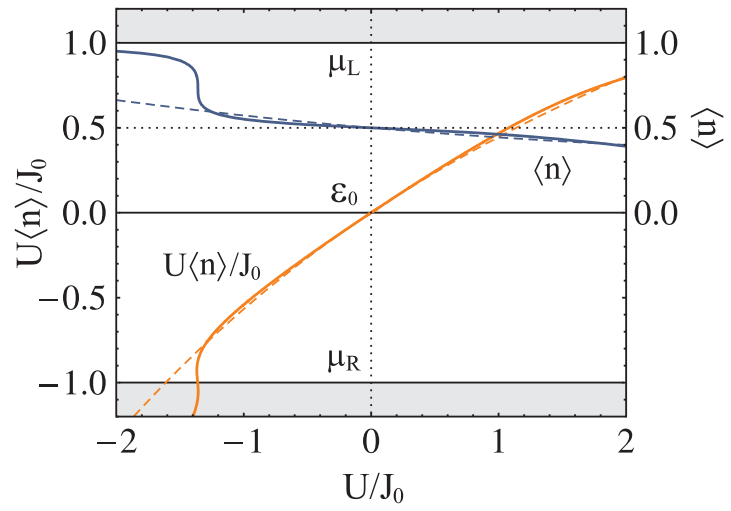

FIG. 5. (Color online) The effect of interactions on a single level with original $(U=0)$ position $\varepsilon_{0}=0$ and the relative chemical potentials $\mu_{L} / J_{0}=1$ and $\mu_{R} / J_{0}=-1$. The occupation $\langle n\rangle$ (blue or dark gray) and the energy shift $U\langle n\rangle / J_{0}$ (orange or light gray) as a function of the interaction $U / J_{0}$ are plotted for the couplings $\Gamma / J_{0}=0.1$ (solid) and $\Gamma / J_{0}=1$ (dashed). For $U>0$ the level is depleted and shifted toward $\mu_{L}$. For $U<0$ the level is almost completely occupied and shifted below $\mu_{R}$ for sufficiently strong interactions. The current is blocked if the single level at $\varepsilon_{0}+U\langle n\rangle$ leaves the energy window $\left[\mu_{L}, \mu_{R}\right]$.

level below $\mu_{R}$. In both cases the single level at $\varepsilon_{0}+U\langle\hat{n}\rangle$ eventually leaves the energy window between $\mu_{L}$ and $\mu_{R}$, and hence the current through the level is strongly suppressed. Thus interactions offer an alternative approach to control the current through the lattice.

\section{CONCLUSIONS}

Using the mesoscopic NEGF-Landauer approach we have analyzed nonequilibrium transport of fermions through an engineered optical lattice for arbitrarily strong coupling to two reservoirs at finite temperatures. We have characterized the full equilibration process by calculating the accumulated number of atoms in the finite reservoirs, which is a directly accessible quantity in experiments. Considering experimentally relevant system parameters we found that the reservoirs equilibrate on time scales comparable to the duration of typical ultracold atom experiments. Our systematic analysis of created and damped fluctuations in the finite system revealed that the mean-field description gives an incomplete picture of fermion transport since significant shot-to-shot variations from the average current, partly due to thermal effects, are to be expected. This is directly relevant to the emulation of semiconductor electronic circuits, where preferably single-shot measurements are required to determine the current [10].

The study of fluctuations around the average current revealed additional information about the processes taking place in the system: We found that thermal fluctuations build up on the time scale of the equilibration process until they reach a constant value proportional to the temperature of the Fermi gas. As an aside, we note that thermal fluctuation between equilibrated reservoirs may therefore be used for thermometry of the system. On a more fundamental level, we saw that a decrease in the current due to a lower transmission of the coherent region is necessarily accompanied by higher quantum fluctuations. This correlation allows the experimenter, e.g., to 
distinguish between changes in either the chemical potentials or the transmission as the cause of a reduced current.

We have shown that modulations of a homogeneous lattice potential can be used not only to reduce the equilibration current, but also to realize a single-level model with full control over the position and the coupling of the level. This setup requires neither additional impurity atoms nor site-bysite manipulations of the optical lattice. Moreover, advanced experimental techniques for producing tailor-made optical potentials, by employing either acousto-optical deflectors [22] or holographic mask techniques [23], are expected to further facilitate the creation of engineered optical lattices. Finally, our mean-field analysis of interaction effects revealed that even weak interactions between the fermions suppress the current through a single level, which can be exploited to control the current.

We conclude with the observation that our approach to nonequilibrium transport between finite reservoirs may be applied to similar ultracold atomic setups [24] or, more generally, to mesoscopic systems such as electrons on liquid helium [25]. Possible extensions of this work include transport of bosonic atoms, similar to the analysis in Refs. [7,26], quantum pumping between reservoirs using time-dependent modulations of the optical lattice [27], and the effect of interactions between fermions on quantum fluctuations [28].

\section{ACKNOWLEDGMENTS}

M.B. thanks Leticia Tarruell for valuable discussions on experimental aspects and Stephen R. Clark for providing clarifying numerical results on nonequilibrium transport. M.B. and W.B. acknowledge financial support from the German Research Foundation (DFG) through SFB 767 and the Swiss National Science Foundation (SNSF) through Project No. PBSKP2/130366.
[1] I. Bloch, J. Dalibard, and W. Zwerger, Rev. Mod. Phys. 80, 885 (2008).

[2] T. Esslinger, Annu. Rev. Condens. Matter Phys. 1, 129 (2010).

[3] G. Roati, E. de Mirandes, F. Ferlaino, H. Ott, G. Modugno, and M. Inguscio, Phys. Rev. Lett. 92, 230402 (2004).

[4] A. Zenesini, C. Sias, H. Lignier, Y. Singh, D. Ciampini, O. Morsch, R. Mannella, E. Arimondo, A. Tomadin, and S. Wimberger, New J. Phys. 10, 053038 (2008).

[5] N. Strohmaier, Y. Takasu, K. Günter, R. Jördens, M. Köhl, H. Moritz, and T. Esslinger, Phys. Rev. Lett. 99, 220601 (2007).

[6] A. Micheli, A. J. Daley, D. Jaksch, and P. Zoller, Phys. Rev. Lett. 93, 140408 (2004).

[7] A. Micheli and P. Zoller, Phys. Rev. A 73, 043613 (2006).

[8] A. J. Daley, S. R. Clark, D. Jaksch, and P. Zoller, Phys. Rev. A 72, 043618 (2005).

[9] R. A. Pepino, J. Cooper, D. Z. Anderson, and M. J. Holland, Phys. Rev. Lett. 103, 140405 (2009).

[10] R. A. Pepino, J. Cooper, D. Meiser, D. Z. Anderson, and M. J. Holland, Phys. Rev. A 82, 013640 (2010).

[11] B. T. Seaman, M. Krämer, D. Z. Anderson, and M. J. Holland, Phys. Rev. A 75, 023615 (2007).

[12] Y. Imry and R. Landauer, Rev. Mod. Phys. 71, S306 (1999).

[13] Y. Nazarov and Y. Blanter, Quantum Transport: Introduction to Nanoscience (Cambridge University Press, Cambridge, 2009).

[14] C. Caroli, R. Combescot, P. Nozieres, and D. Saint-James, J. Phys. C 4, 916 (1971).
[15] J. C. Cuevas and E. Scheer, Molecular Electronics: An Introduction to Theory and Experiment (World Scientific Publishing Company, Singapore, 2010).

[16] A. Griessner, A. J. Daley, S. R. Clark, D. Jaksch, and P. Zoller, Phys. Rev. Lett. 97, 220403 (2006).

[17] C. W. Gardiner, Handbook of Stochastic Methods for Physics, Chemistry and the Natural Sciences, 3rd ed. (Springer-Verlag, Berlin, 2004).

[18] The energy levels $E_{\ell}$ of the uncoupled coherent part $C$ with constant hopping $J_{0}$ are given by $E_{\ell}=2 J_{0}-2 J_{0} \cos [\pi \ell /(m+$ 1)] with $\ell=1,2, \ldots, m$.

[19] R. Grimm, M. Weidemüller, and Y. B. Ovchinnikov, Adv. At. Mol. Opt. Phys. 42, 95 (2000).

[20] P. W. Anderson, Phys. Rev. 124, 41 (1961).

[21] W. von der Linden, I. Morgenstern, and H. de Raedt, Phys. Rev. B 41, 4669 (1990).

[22] B. Zimmermann, T. Müller, J. Meineke, T. Esslinger, and H. Moritz, New J. Phys. 13, 043007 (2011).

[23] M. Pasienski and B. DeMarco, Opt. Express 16, 2176 (2008).

[24] B. Gadway, D. Pertot, J. Reeves, and D. Schneble, e-print arXiv:1104.2564.

[25] D. G. Rees, I. Kuroda, C. A. Marrache-Kikuchi, M. Höfer, P. Leiderer, and K. Kono, Phys. Rev. Lett. 106, 026803 (2011).

[26] C.-C. Chien, M. Zwolak, and M. Di Ventra, e-print arXiv:1110.1646.

[27] K. K. Das and S. Aubin, Phys. Rev. Lett. 103, 123007 (2009).

[28] D. Kambly, C. Flindt, and M. Büttiker, Phys. Rev. B 83, 075432 (2011). 